\title{
Cognitive Impairments and the Prevention of Homelessness: Research and Practice Review
}

\author{
Thomas Earl Backer · Elizabeth A. Howard
}

Published online: 5 June 2007

(C) Springer Science+Business Media, LLC 2007

\begin{abstract}
Cognitive impairments can increase significantly a person's level of risk for becoming or remaining homeless. Five topics are explored that address these impairments in preventing homelessness: (1) the nature and estimated prevalence of cognitive impairments among people who are homeless, especially those with vulnerabilities like mental illness or substance abuse; (2) the multiple origins of these impairments; (3) how these impairments impact services for people at risk for homelessness; (4) good practice approaches to handling cognitive impairments in homeless shelters, supported housing programs, and other service systems for people at risk for homelessness; and, (5) important research and practice issues requiring further action.
\end{abstract}

Keywords Cognitive impairments · Homelessness · Prevention ·

Assessment · Intervention

\section{Introduction}

Cognitive impairments can significantly affect an individual's ability to obtain and maintain stable housing, and to benefit from supportive services. Deficits in a person's memory, perception, judgment, planning, and speech can result in poor problem-solving and social skills, and in his/her inability to make sound decisions. The behaviors resulting from cognitive impairments are largely unintentional, but they are often poorly understood by providers and others, and are sometimes mistaken for noncompliance. For example, landlords sometimes deny housing to people with cognitive impairments because their behaviors may be difficult to understand. Frequently behaviors that occur as a result of cognitive impairments are misinterpreted as being intentionally antisocial or disruptive. People with

T. E. Backer $(\bowtie) \cdot$ E. A. Howard

Human Interaction Research Institute, 5435 Balboa Boulevard, Suite 115, Encino, CA 91316, USA e-mail: hirila@aol.com 
cognitive impairments who do obtain housing often lose it through eviction, because these impairments leave them unable to figure out how to pay for rent and utilities, or how to maintain their residences appropriately (HCH Clinicians' Network 2003).

The nature, prevalence, and origins of cognitive impairments are relevant to the prevention of homelessness for all three levels of "prevention" as classically defined. Primary prevention refers to preventing people who are at risk but not yet homeless from becoming so, and might involve providing cognitive remediation or other services more widely. Secondary prevention and tertiary prevention activities focus on helping people who already are homeless (especially the core population of chronically homeless people) find permanent and stable housing. The good practices presented here for assessing and intervening with cognitive impairments are specific to secondary or tertiary prevention services provided through homeless shelters, supported housing programs, and other service programs for people who already have had some experience with homelessness.

At present only a limited research and practice literature is available to support families and mental health care workers in finding solutions for people with cognitive impairments at risk for homelessness (Spence et al. 2004). In many communities, "neuropsychological assessments, diagnosis and treatment for homeless people tend to be inadequate or altogether lacking"' (Solliday-McRoy et al. 2004, p 471). This review examines five questions related to these challenges: (1) What are cognitive impairments and how many homeless people are cognitively impaired? (2) What are the origins of these cognitive impairments? (3) How do cognitive impairments impact services for people at risk for homelessness? (4) What are some good practice approaches to handling cognitive impairments in homeless shelters, supported housing programs, and other service systems for people at risk for homelessness? and (5) What research and practice issues require further action?

Gladwell (2006) describes the high costs of homelessness for people who are extremely vulnerable. Costs might be reduced by using a "power law" approach, increasing impact of services and cost-effectiveness by giving priority to the most severely disabled people. If the presence of significant cognitive impairments is understood to be "severe," people with these severe disabilities would be candidates for enhanced services under a "power law' -type policy. This approach is controversial because it tends to pit subgroups of people who are homeless against each other. However, this is a policy option requiring consideration, given tight resources.

The National Alliance to End Homelessness believes that ending chronic homelessness is well within the nation's grasp and could be accomplished within 10 years given public will and funding support (National Alliance to End Homelessness 2000). Recently, the Department of Housing and Urban Development announced $\$ 1.2$ billion in funding for homeless assistance (National Alliance to End Homelessness 2005). This funding underscores a growing commitment among funders, communities, advocates, and service providers to deal more systemically with chronic homelessness. Addressing cognitive impairments among people at risk for homelessness can enhance the larger systems change effort, as well as individual person-focused efforts to prevent homelessness. 


\section{What are Cognitive Impairments and How Many Homeless People are Cognitively Impaired?}

At least 20 million Americans suffer from some type of mild to severe cognitive impairment. These impairments result from brain disease or injury and compromise "perception, information processing or formulation of a verbal or motor response", (HCH Clinicians' Network 2003). They affect attention, memory, and language; they have impact on visual and spatial functioning, the ability to perform unfamiliar tasks, overall awareness, and the ability to organize and regulate behavior (Filley 2002).

It is difficult to estimate precisely the number of people with cognitive impairments in the homeless population. People in shelters are infrequently assessed for cognitive impairments. Even when they are, they are often treated only for other conditions, such as severe and persistent mental illness or substance abuse. For people on the streets, the ability to assess cognitive impairments may be compromised by more temporary conditions, such as inebriation or recent drug use.

Spence et al. (2004) reviewed published work to assess evidence of cognitive dysfunction among adults who are homeless. Despite liberal inclusion criteria, they identified only 17 studies, conducted mainly in the U.S. with about 3,300 subjects in all. These studies indicate that there is a considerable burden of cognitive dysfunction among homeless people, although existing research does not directly establish a causal connection.

Solliday-McRoy et al. (2004) estimate that the prevalence of cognitive impairment among people who are homeless may be as high as $80 \%$. Douyon and colleagues (1998) found that homeless veterans displayed higher levels of cognitive impairments than non-homeless individuals. They also showed higher levels of hostility, prior criminal activity, and family history of psychiatric illnesses, but lower levels of depression. This study identified practical consequences of leaving cognitive impairments undiagnosed and untreated. For instance, if higher hostility is common among homeless people with cognitive impairments, it can affect their ability to access and sustain service involvement.

\section{What are the Origins of These Cognitive Impairments?}

Cognitive impairments arise from a number of sources-including schizophrenia, substance abuse, traumatic, or acquired brain injury, progressive neurological disorders, and developmental disabilities. For many homeless people, their impairments may come from more than one source, and the pattern of their cognitive problems may shift over time. Each of these sources is discussed below.

Cognitive impairment is a core feature of schizophrenia, affecting at least $85 \%$ of this population (Gopal and Variend 2005; Keefe and Hawkins 2005), with resulting negative impact on the ability to maintain stable housing and otherwise function effectively in the community. These difficulties can be significantly reduced with appropriate medication, in concert with cognitive remediation therapy (Gopal and Variend 2005; Wexler and Bell 2005), which is discussed below. 
Poor attention is an early cognitive symptom, but difficulties with memory and visual motor speed may also be present, even before the onset of psychotic symptoms (Medalia and Revheim 2002). People with schizophrenia also seem unable to maintain voluntary control over the maintenance and manipulation of information (Goldberg and Green 2002), so they may have difficulty formulating plans, implementing them, and then flexibly changing to a new strategy once an old one no longer works. Research typically shows abnormalities in both the frontal and temporal lobes of their brains, controlling respectively the executive/attentional systems and memory systems (Rains et al. 1995). In severe cases, people who have such impairments often cannot complete daily activities without assistance (Rains et al. 1995; Turetsky et al. 2002).

Prolonged substance abuse may result in more temporary and reversible cognitive impairments. Delirium often results from severe intoxication, and impairments from abuse of drugs such as cocaine also are common (Osher 2006, personal communication). Because many people at risk for homelessness are substance abusers, interventions that reduce substance use can also reduce these temporary cognitive impairments, and sometimes the more enduring ones described below. This in turn can play a significant role in efforts to prevent homelessness (National Alliance to End Homelessness 2000).

Traumatic brain injury resulting in cognitive impairments is seen more frequently in people who are homeless than in the general population (Gonzalez et al. 2001; HCH Clinicians' Network 2003). Traumatic brain injury may be caused by auto accidents, falls, accidental poisonings, assaults, or gunshot wounds. Homeless individuals bear a disproportionate risk for severe head injury, at least partly because they are more likely to be victimized by assaults, have higher propensity for risk taking, and have a higher prevalence of antisocial personality disorder (Silver and Felix 1999).

Acquired brain injury most often occurs among people who are homeless as a result of chronic substance abuse, strokes, post-traumatic stress disorder (particularly likely among the many homeless people who are veterans), and infections or malnutrition (Silver and Felix 1999). All these types of injuries may have been acquired as a consequence of being homeless.

Other cognitive impairments are associated with progressive neurological disorders. These include multiple sclerosis, Parkinson's, and Alzheimer's disease or dementia due to aging (HCH Clinicians' Network 2003).

Developmental disabilities are a diverse group of severe chronic conditions that are due to mental and/or physical impairments, resulting in problems with major life activities such as language, mobility, learning, self-help, and independent living. Developmental disabilities begin anytime during development up to 22 years of age, and usually last throughout a person's lifetime. They are attributable to such conditions as mental retardation, cerebral palsy, or autism, and typically involve some degree of cognitive impairment. Nearly four million of the estimated 20 million Americans with cognitive impairments have a developmental disability (U.S. Department of Health and Human Services 2000). Because developmental disabilities are typically diagnosed early in childhood, many assessment and treatment protocols for these impairments are offered as part of 
special education programs. Early intervention services are designed to meet developmental needs of an infant or toddler, and then lead to special education programs for school-age children (National Dissemination Center for Children with Disabilities 2003).

Other sub-types of developmental disabilities, typically diagnosed in very young children, are the various conditions covered by the umbrella term Fetal Alcohol Spectrum Disorder (FASD), all resulting from a mother's alcohol abuse during pregnancy. Over half of all children diagnosed with these conditions are clinically defined as having attention deficits, resulting in significant impact on their daily lives, as children and later as adults (Lockhart 2001).

As many as $35 \%$ of people with a developmental disability also have a mental illness. Prevalence rates for this kind of dual diagnosis are unreliable because individuals diagnosed with developmental disabilities usually are not assessed for mental illness. People with co-occurring mental illness and developmental disabilities rarely receive appropriate assessment and care for both disorders. As a result, they may be at increased risk for long-term institutionalization and homelessness (National Association of State Mental Health Program Directors 2004).

People with developmental disabilities are more vulnerable to becoming homeless at two critical junctures. The first occurs when a young person ages out of the care system designed for children (including, in many cases, foster care). Special education and other in-school programs can no longer offer services, and adult supports may not be accessible (National Dissemination Center for Children with Disabilities 2003). Unless the individual has strong family ties, they are at high risk of becoming homeless. The second juncture occurs when parents of a developmentally disabled person are no longer living. If plans for support and stable housing are not in place before the death of the parents, the individual is more likely to become homeless.

\section{How do Cognitive Impairments Impact Services for People at Risk for Homelessness?}

Assessing cognitive impairments

Various instruments are available for assessing the presence and severity of cognitive impairments. For example, in a review conducted by Spence et al. (2004), 10 of the 18 agencies studied employed the Mini-Mental Status Examination (MMSE) to assess cognitive functioning - a widely-used but relatively non-specific assessment tool (Gonzalez et al. 2001). The Cognitive Impairment Assessment Education Series (Weaverdyck 2006), like the MMSE, provides questions in a format that service agency staff who are not mental health professionals can use to assess cognitive functioning. Instruments commonly used by mental health professionals also include the Repeatable Battery for the Assessment of Neuropsychological Status (RBANS) and the Brief Assessment of Cognition in Schizophrenia (BACS) (Keefe and Hawkins 2005). 
Inspite of the availability of assessment instruments, cognitive impairments of people who are homeless often are not assessed, (Osher 2006; Solliday-McRoy et al. 2004). Service workers are often focused on addressing pressing survival needs of people who are homeless, such as food, shelter, and medical attention $(\mathrm{HCH}$ Clinicians' Network 2003). Furthermore, shelter staff and others serving people who are homeless are often untrained in assessment.

When assessments are conducted, they can be quite challenging (Eberlein 2000). Individuals with cognitive impairments may be unable to participate fully in assessment because of their inability to communicate their thoughts, experiences, and feelings (Fodero n.d.). According to Eberlein (2000), cognitively impaired individuals often find it difficult to undergo formal neuropsychological assessment, and sometimes may be reluctant to do so, even though it may provide helpful diagnostic information. Later, as they receive services, people with cognitive impairments may be unable to discuss their discomfort with service protocols (sometimes severe enough to cause them to reject services), rules for living in a shelter or supported housing program, or difficulties such as medication side effects (Benson and Wilkerson 2000).

Misdiagnosis may also be a problem, especially due to the multiple causes of cognitive impairments. For example, people with severe cognitive impairments that are not related to a mental illness such as schizophrenia are still sometimes diagnosed only as mentally ill, resulting in incomplete or inappropriate services (HCH Clinicians' Network 2003). For example, antipsychotic medications will only ameliorate cognitive impairments that were caused by schizophrenia.

Other health issues may complicate assessment of cognitive impairments. Symptoms of some diseases can mimic organic brain disorders. For example, cognitive impairment in a homeless person with HIV may indicate AIDS-related dementia, but it can also indicate prolonged depression, opportunistic infection, side effects from medication, or self-medication with psychoactive substances. Confusion, incoherence, and distorted speech can indicate cognitive impairment, but it may be related to untreated diabetes; people with pre-lingual deafness are sometimes diagnosed as psychotic or mentally retarded ( $\mathrm{HCH}$ Clinicians' Network 2003).

The reason for undertaking assessment is not to attach a label, but rather to identify areas of need and services required if cognitive impairments are present (Benson and Wilkerson 2000). In practical terms, however, a diagnostic label may be useful if it allows the individual to qualify for needed services. For instance, full neuropsychological testing may reveal that an individual qualifies for services from the adult developmental disabilities system (Osher 2006), or for SSI/SSDI benefits.

\section{Serving People with Cognitive Impairments}

People with major cognitive impairments are often among the most challenging to serve in programs that focus on people at risk for homelessness, such as shelters or supported housing programs (HCH Clinicians' Network 2003). Cognitive impairments may be experienced in many different ways; they may impact an individual's 
ability to function in one area, but not in others. Some people can appear high functioning when engaged in social or casual conversation, but are nevertheless impaired to an extent that increases their risk for becoming or remaining homeless (Medalia and Ravjeo 2002).

Cognitively impaired people may be erroneously considered "non-compliant" or "in denial" because their impairments preclude their full participation in the service process (Gonzalez et al. 2001). Sometimes they are banned from service settings due to impairment-related disruptive behavior or failure to comply with prescribed treatments (HCH Clinician's Network 2003). Folk and Fernandez (n.d.) give the example of a woman with undiagnosed mild mental retardation who was constantly in danger of being kicked out of a shelter because she did not do her chore of vacuuming the floor at night. She always would agree to the task when asked by staff, but then did not follow through. This led them to assume she was manipulative and resistant. An advocate who suspected she might be cognitively impaired discovered that she did not know how to operate a vacuum cleaner-but agreed to do so when asked to please the shelter workers.

Cognitively impaired people may reject services, sometimes rebuffing even the most basic interactions with service staff. This may happen because they are unable to comprehend what is being asked, or the benefit from their participation. Such people also may resist services because they have fixed and inaccurate ideas about the service system. This situation is only made more complicated by cognitive impairments that affect their ability to judge and understand. In some cases, the best providers can do is to keep these individuals from leaving the service system altogether, and significantly worsening their life situations ( $\mathrm{HCH}$ Clinicians' Network 2003). Whenever the service system seems to be "failing" someone, service staff can check again to determine whether an undiagnosed cognitive impairment is contributing to the individual's difficulties.

People with cognitive impairments also may be more vulnerable to substance abuse because of an inability to regulate their behavior (Price 2005). These individuals often lack protective and resiliency factors (e.g., feelings of selfcompetence, self-control, and personal power) that can protect against the temptation to abuse substances. They may self-medicate against feelings of loss and frustration specific to their cognitive functioning. Using drugs or alcohol in a social context may also help to reduce the social isolation resulting from their impairment.

Moreover, people with co-occurring cognitive impairments and substance abuse are often unable to benefit from or participate in traditional substance abuse treatment programs. Some programs move too quickly, or require a level of intellectual participation cognitively impaired people may find difficult (such as some aspects of 12-step programs). If the impairment is severe, the person may not understand the effects of substance abuse, or the workings of a particular program, but counselors may interpret this as denial (HCH Clinicians' Network 2003). Counselors may lack the skills to respond to people with cognitive impairments and make needed adjustments in the service process.

For instance, motivational interviewing is an evidence-based practice designed for engaging ambivalent or resistant people (Squires and Moyers 2001). It relies 
heavily on an individual's ability to participate in an open exchange with others. It assumes a level of self-sufficiency and judgment that simply is not present in many people with substantial cognitive impairments. Thus, despite its powerful evidence base, providers may need to find alternatives to motivational interviewing when they have concerns about a person's level of cognitive impairment.

Many juveniles and adults with cognitive impairments become involved with the criminal justice system, but their disabilities may not be easily recognized by intake personnel. Arrestees may not understand their rights, and frequently waive them. Once in jail or prison, other inmates often victimize people with cognitive impairments. They may spend long periods in segregation because they have difficulty understanding jail and prison rules, or orders from guards. Because they often are not identified as cognitively impaired, and because there are few specialized programs for reintegrating people with these disabilities after they are released, they often are discharged to homelessness, and thus enter "revolving door cycles" like prison to parole and back to prison (Petersilia 2000). In addition, they may be refused housing because of their criminal records.

Services also need to respond to ethnic and cultural differences in conceptualizing cognitive impairments, as well as to people's abilities to understand and participate in their service programs (National Association of State Mental Health Program Directors 2004). To deal with these challenges in the Seattle area, the Arc of King County's program for homeless people who have developmental disabilities (described below) offers cultural competency training for both service recipients and professionals (The Arc of King County 2006).

\section{What are Some Good Practice Approaches to Handling Cognitive Impairments in Homeless Shelters, Supported Housing Programs, and Service Systems for People who are Homeless or at Risk for Homelessness?}

Strategies for handling cognitive impairments among people at risk for homelessness exist, but are not well-integrated into standard practice because they come from varied settings and disciplines. Space does not permit a more detailed examination of these approaches, but listed below are some representative good practices. They include (a) general strategies for providing services, (b) services that target people with identified cognitive impairments, and (c) methods for training service providers or family members.

Solliday-McRoy et al. (2004) analyzed research- and practice-based strategies for modifying assessment, treatment, or shelter/supported housing services to reflect the special needs of people with significant cognitive impairments. They suggest the following general strategies, and claim they can be useful regardless of the source of the impairment:

- Use service modalities that are not cognitively demanding,

- Set well-defined goals for all services,

- Repeat all basic concepts and instructions,

- Provide concrete explanations of actions to be taken, 
- Offer continual behavioral reinforcement,

- Use memory enhancement approaches,

- Use cognitive rehabilitation approaches when feasible, and

- Use structured skill learning as part of services.

When working with people with cognitive impairments, it is important to use very clear, concise, and specific language, avoiding abstractions and unnecessary detail. Information should be provided slowly, with opportunity for questions. Goals can be broken down to one-at-a-time, instead of presenting the person with a set of goals they may find overwhelming. Although adults with cognitive impairments may at time appear childish, they are adults and should be treated as such. It may be necessary to repeat instructions frequently, use diagrams or demonstrations, and ask the person to repeat instructions (HCH Clinicians Network 2003).

Programs offered by a shelter or other service agency can serve people with cognitive impairments more effectively by adapting one or more of the above general approaches. For example, medication management programs can be custom-fitted for people in supported housing programs who have cognitive impairments. This may include increasing the time allotted for explanations or using photographs or pictures from journals or magazines to illustrate instructions (Osher 2006).

Some service programs by their nature are more amenable to such custom-fitting. For instance, many homeless health care clinics use intensive case management approaches based on Assertive Community Treatment (ACT), an evidence-based approach to intensive services (Lehman et al. 1999). The person is fully engaged in treatment from the beginning, and sets goals such as stable housing, employment, and self-sufficiency. Under these conditions, cognitive impairments, even if not formally diagnosed, are more likely to be recognized and can be dealt with in the service process.

Velligan and colleagues (2006) promote custom-fitting of another type. Considerable research shows that "cognitive adaptation training", with individually-tailored environmental supports (signs, alarms, special medication containers, etc.) can help people with medication compliance and other adaptive behaviors. However, in a controlled study Velligan et al. (2006) found that such supports are not likely to be used unless they are customized to meet the needs of individuals, and set up in their own home environments.

"'Errorless learning,', used successfully in teaching entry-level job tasks, also is effective in teaching social problem-solving skills to people with schizophrenia (Kern et al. 2005). This approach, which breaks down task performance into smaller, more-easily-mastered subunits, may have potential for application to other kinds of learning needs for people with cognitive impairments, whether arising from schizophrenia or not.

Harvey et al. (2005) suggest that since cognitive impairment is a major determinant of functional outcome in schizophrenia, treatment of cognitive impairment at the time of the first psychotic episode might have the potential to change functional outcomes of the illness. Their study examined changes in cognitive functioning associated with treatment with risperidone compared with 
haloperidol. Treatment with risperidone at the time of the first episode of schizophrenia is associated with wide-ranging improvements in cognitive functioning; overall improvement is significantly greater with risperidone than with haloperidol. Thus, treatment decisions related to a primary diagnosis of schizophrenia can have an impact on reducing cognitive impairments, in cases where mental illness was the primary source of the impairment.

Cognitive remediation is an intervention for improving cognitive function, which has shown large and lasting improvements in cognition for people with schizophrenia (Wexler and Bell 2005); it may also have potential for handling cognitive impairments of all types. Exercises to improve attention, memory and executive function are performed in either individual or group settings, sometimes with computerized exercises or manualized programs. Growing evidence suggests that cognitive remediation improves real-world functioning as well (Gaddes and Edgell 1994; Medalia and Richardson 2005).

Comprehensive service programs have been developed to address the needs of people with cognitive impairments who are at risk for homelessness. For instance, the Arc of King County Survival Services Program (The Arc of King County 2006) has since 1989 provided services specifically for people who are homeless with developmental disabilities. Survival Services helps people secure and maintain public assistance, locate affordable housing, and provides crisis intervention on an ongoing basis. The aim is to teach people the skills necessary to achieve the highest possible level of personal responsibility.

The Homeless Case Management Program in Silver Spring, Maryland, published a resource document for providers serving people who are homeless and have developmental disabilities (Folk and Fernandez n.d.). The Maryland Developmental Disabilities Administration (2006) provides an array of services to individuals in crisis, including those at high risk for homelessness. Services help adults deal better with their cognitive impairments, and continue living in their own homes or with their families, or in a residential service setting. Many other states have similar programs, such as the New York Office of Mental Retardation and Developmental Disabilities (2006) and its Options for People Through Services (OPTS) program.

Service programs can be enhanced by bringing together agencies and professionals from mental retardation/developmental disabilities and mental health to design programs and treatment protocols that will address both cognitive impairment and mental illness (as well as substance abuse, when needed). This is particularly important because public services often are organized as though individuals have only one disability, e.g., mental illness or developmental disability, whereas in fact they may have several (Shriver 2001). In a report on a conference bringing together these two systems, the National Association of State Mental Health Program Directors (2004) provide a number of suggestions for how a more integrated approach could be developed, with resulting improvements in services for people who are homeless.

In many service settings, staff are not trained to detect or respond to cognitive impairments in people who are homeless, often because of limited financial resources, or simply because training is not available. The need is particularly acute 
for training to serve people with multiple challenges (mental illness, substance abuse, developmental disabilities, etc). The Ohio Coordinating Center of Excellence (CCOE) in Dual Diagnosis trains professionals in mental health/mental retardation/ developmental disabilities and related service systems (including but not limited to those focused on people at risk for homelessness). It also provides clinical and organizational consultation across Ohio. CCOE developed regional training programs where systems change and clinical best practices are presented, and provided mini-grants to foster local collaboration, as well as offer individual consultation to clinicians (Benson and Wilkerson 2000).

Medalia and Revheim (2002) assembled a handbook for families and friends of individuals with psychiatric disorders, to help them understand and respond to cognitive impairments of these individuals. It discusses the impact of cognitive impairment on daily functioning and outlines various treatment and service options. The manual also discusses things family members can do to help the individual improve his/her cognitive functioning.

\section{What Research and Practice Issues Require Further Action?}

More research is needed to document the prevalence of cognitive impairment among people who are homeless and at risk for homelessness, about the origins and severity of their impairments, and about the consequences of these impairments for the effectiveness of the service process. Study also is needed about how to economically and accurately assess cognitive impairments. For instance, considerable empirical evidence indicates that cognitive impairments, when properly assessed, are among the most important predictors of long-term disability in schizophrenia. The MATRICS project, funded by the National Institute of Mental Health, is developing more rigorous assessment methods, along with pharmacological approaches to cognitive impairments among people with schizophrenia, and ways to encourage the pharmaceutical industry to develop promising drugs for this purpose (MATRICS Project 2006).

Controlled studies are essential to determine more precisely the effectiveness of innovative methods or service programs that address cognitive impairments among people in service systems, including supportive housing and homeless shelters. Of particular value would be research showing that cost savings are likely when cognitive impairments are addressed more systematically among people who are homeless, as well as studies about how to use these approaches in primary prevention of homelessness - among populations with cognitive impairments who are not yet homeless.

Re-shaping of public policy might be possible based on good cost-benefit data. Also, more research is needed on newer types of preventive interventions, such as drugs that reduce the disabling effects of cognitive impairment (Filley 2002). Neurosurgical techniques have advanced, and may lead to helpful interventions in the future. There is some hope that gene therapies may be created that can help treat some of these impairments. Also, the University of Colorado's Coleman Institute 
for Cognitive Disabilities (2006) is pioneering the development of technology to help individuals residing in community living environments. When more widely implemented, these technologies may reduce the risk of homelessness. All of these approaches are still in the early stages of development.

At the practice level, mental health, substance abuse and mental retardation/ developmental disability professionals need to continue working together to develop integrated assessment and service programs. Medical professionals might be included, as well, since homeless people are at high risk for injury, and for diseases such as HIV/AIDS, which can also result in cognitive impairments. The National Association of State Mental Health Program Directors conference report (2004) provides a number of concrete recommendations for such service and systems integration.

Stigma remains an issue for many people in the homeless service system-many people will not come into the service system because they fear the negative effects of stigma. Misconceptions and prejudices of providers about people with cognitive impairments are part of the challenge. Professional as well as public education is needed, to promote greater awareness of the special challenges faced by people with cognitive impairments who are homeless or at risk for homelessness. In general, more resources are needed to support training of staff and improvement of service systems so they can better respond to the needs of people with cognitive impairments.

Lastly, a Federal interagency task force needs to be assembled to examine the complex issues of cognitive impairments and prevention of homelessness. This task force could include representatives from various Federal agencies concerned with mental illness and substance abuse, as well as those concerned with homelessness. It could include researchers, providers, foundation representatives, policymakers, and people at risk for homelessness and their family members. Such a task force could spearhead development of a toolkit on assessment and treatment of cognitive impairments for people who are at risk of or experiencing homelessness. Such a toolkit could then be disseminated widely, as part of a national plan for workforce development on this subject.

Action on all of the issues just outlined will be needed to answer the five questions that framed this review of research and practice on cognitive impairments. These challenges are a fact of life for many people who are homeless or at risk for homelessness, and for service providers, their families, and the community at large. The difficulty of creating good practices is increased because these impairments have multiple origins, and they are not easy to diagnose or treat. However, progress on the several fronts discussed here is essential, if we hope to end chronic homelessness in the United States within the next ten years.

Acknowledgments The authors acknowledge with gratitude the input provided by Dr. Deborah Stone, Center for Mental Health Services; and Dr. Mary Anne Myers, Westat. Useful input also was provided by Dr. Fred Osher, University of Maryland; William Compton, Project Return, Los Angeles; Dr. Alex Kopelowicz, San Fernando Mental Health Services; Dr. Timothy Kuehnel, research psychologist; and Daryl Washington, Vanessa Heim and Temma Allen, all of the Fairfax County Community Services Board. Preparation of this paper was supported by the Knowledge Application Program Project, Center for Mental Health Services Task Order 270-03-6005. 


\section{References}

The Arc of King County. (2006). Survival services program. Seattle, WA: Author.

Benson, B., \& Wilkerson, D. (2000). Report and recommendations: Clinical best practices for serving people with developmental disabilities and mental illness. Columbus, $\mathrm{OH}$ : Department of Mental Health/Depertment of Mental Retardation and Developmental Disabilities Advisory Committee.

Coleman Institute for Cognitive Disabilities. (2006). Coleman Institute sponsored projects. University of Colorado, Retrieved February 12, 2007, from http://www.colemaninstitute.org.

Douyon, R., Guzman, P., Romain, G., Ireland, S. J., Mendoza, L., Lopez-Blanco, M., et al. (1998). Subtle neurological deficits and psychopathological findings in substance-abusing homeless and nonhomeless veterans. Journal of Neuropsychiatry and Clinical Neurosciences, 10, 210-215.

Eberlein, L. (2000). Guidelines for the evaluation of persons dually diagnosed with mental retardation and mental illness. In B. Benson \& D. Wilkerson (Co-Chairs) Report and recommendations: Clinical best practices for serving people with developmental disabilities and mental illness (pp. 6-12). Columbus, OH: Ohio Department of Mental Health/Ohio Development of Mental Retardation and Developmental Disabilities Advisory Committee.

Filley, C. M. (2002). Cognitive disorders: The state of the science. Coleman Institute for Cognitive Disabilities, University of Colorado School of Medicine, Unpublished manuscript.

Folk, L., \& Fernandez, C. B. (Eds.) (no date). Helping homeless people with developmental disabilities: A guide for providers. Rockville/Silver Spring, MD: Affiliated Sante Group and The Lt. Joseph P. Kennedy Institute.

Fodero, J. (no date). Forgotten: The other dually diagnosed. Retrieved February 12, 2007, from http:// intotem.buffnet.net $/ \mathrm{mhw} / 30 \mathrm{jf} . \mathrm{html}$.

Gaddes, W. H., \& Edgell, D. (1994). Learning disabilities and brain function: A neuropsychological approach. (3rd ed.) New York: Springer-Verlag.

Gladwell, M. (2006, February 13 \& 20). Million-dollar Murray. The New Yorker, 96-107.

Goldberg, T. E., \& Green, M. F. (2002). Neurocognitive functioning in patients with schizophrenia: An overview. In K. L. Davis, D. Charney, J. T. Coyle \& C. Nemeroff (Eds.), Neuropsychopharmacoogy: The fifth generation of progress (pp. 657-670). Nashville, TN: American College of Neuropsychopharmacology.

Gonzalez, E. A., Dieter, J. N., Natalie, R. A., \& Tanner, S. L. (2001). Neuropsychological evaluation of higher-functioning homeless persons: A comparison of an abbreviated test battery to the MiniMental State Exam. The Journal of Nervous and Mental Disease, 189(3), 176-181.

Gopal, Y. V., \& Variend, H. (2005). First-episode schizophrenia: Review of cognitive deficits and cognitive remediation. Advances in Psychiatric Treatment, 11, 38-44.

HCH Clinicians' Network. (2003). Dealing with disability: Cognitive impairments \& homelessness. Nashville, TN: HCH Clinicians' Network.

Harvey, P. D., Rabinowitz, J., Eerdekens, M., \& Davidson, M. (2005). Treatment of cognitive impairment in early psychosis: A comparison of risperidone and haloperidol in a large long-term trial. American Journal of Psychiatry, 162, 1888-1895.

Kern, R. S., Green, M. F., Mitchell, S., Kopelowicz, A., Mintz, J., \& Liberman, R. P. (2005). Extensions of errorless learning for social problem-solving deficits in schizophrenia. American Journal of Psychiatry, 162, 513-519.

Keefe, R., \& Hawkins, K. (2005). Assessing and treating cognitive deficits. Psychiatric Times, 23(3), 1-4.

Lehman, A. F., Dixon, L., Hoch, J. S., Deforge, B., Kernan, E., \& Frank, R. (1999). Cost-effectiveness of assertive community treatment for homeless persons with severe mental illness. British Journal of Psychiatry, 174, 346-352.

Lockhart, P. J. (2001). Fetal alcohol spectrum disorders for mental health professionals: A brief review. Current Opinions in Psychiatry, 14, 463-469.

Maryland Developmental Disabilities Administration. (2006). Services provided by DDA. Retrieved February 12, 2007, from http://www.ddamaryland.org.

MATRICS Project (2006). Overview. Retrieved February 12, 2007, from http://www.matrics.ucla.edu.

Medalia, A., \& Revheim, N. (2002). Dealing with cognitive dysfunction associated with psychiatric disabilities: A handbook for families and friends of individuals with psychiatric disorders. Albany, NY: New York State Office of Mental Health.

Medalia, A., \& Richardson, R. (2005). What predicts a good response to cognitive remediation interventions? Schizophrenia Bulletin, 31(4), 942-953. 
National Alliance to End Homelessness. (2005). Comparing proposals for HUD McKinney-Vento funding. Washington, DC: National Alliance to End Homelessness.

National Alliance to End Homelessness. (2000). A plan not a dream: How to end homelessness in ten years. Washington, DC: National Alliance to End Homelessness.

National Association of State Mental Health Program Directors. (2004). Serving individuals with cooccurring developmental disabilities and mental illnesses: Systems barriers and strategies for reform. Alexandria, VA: National Association of State Mental Health Program Directors.

National Dissemination Center for Children with Disabilities. (2003). Pervasive developmental disorders: Fact sheet 20 (updated from 1998). Washington, DC: National Dissemination Center for Children with Disabilities.

New York Office of Mental Retardation and Developmental Disabilities. (2006). Options for people through services: General information brochure. Retrieved February 20, 2007, from http:// www.omr.state.ny.us.

Petersilia, J. (2000). Doing justice? Criminal offenders with developmental disabilities. California Policy Research Center Brief Series, 12(4), 1-62.

Price, A. (Ed.) (2005). The intersection of developmental disabilities, substance abuse and parenthood: Challenge and response. The Source, 14(2), pp. 1-4.

Rains, G. D., Sauer, K., \& Kant, C. (1995). Cognitive impairment consistent with left frontal-temporal abnormality in schizophrenia patients. Journal of the International Neuropsychological Society, 1(2), 133.

Shriver, E. (2001). Presentation on behalf of the Joseph P. Kennedy Foundation (no title) to workshop convened by the National Institute of Neurological Disorders and Stroke, the National Institute of Child Health and Human Development, the National Institute of Mental Health, the National Institute of Health Office of Rare Diseases and the Joseph P. Kennedy Foundation.

Silver, J. M., \& Felix, A. (1999). Neuropsychiatry and the homeless. In F. Ovsiew (Ed.), Neuropsychiatry and mental health services (pp. 319-333). Washington, DC: American Psychiatric Press.

Solliday-McRoy, C., Campbell, T. C., Melchert, T. P., Young, T. J., \& Cisler, R. A. (2004). Neuropsychological functioning of homeless men. Journal of Nervous and Mental Disease, 192(7), $471-478$.

Spence, S., Stevens, R., \& Parks, R. (2004). Cognitive dysfunction in homeless adults: A systematic review. Journal of the Royal Society of Medicine, 97, 375-379.

Squires, D. D., \& Moyers, T. B. (2001). Motivational interviewing: A guideline developed for the Behavioral Health Recovery Management Project. Springfield, IL: Illinois Department of Human Services, Office of Alcoholism and Substance Abuse.

Turetsky, M. D., Moberg, P. J., Mozley, L. H., Moelter, S. T., Agrin, R. N., Gur, R. C., et al. (2002). Memory-delineated subtypes of schizophrenia: Relationship to clinical, neuroanatomical, and neurophysiological measures. Neuropsychology, 16(4), 481-490.

U.S. Department of Health, Human Services, Administration for Children and Families (2000). Federal developmental disabilities programs. Washington, DC: U.S. Department of Health and Human Services, Administration for Children and Families.

Velligan, D. I., Mueller, J., Wang, M., DiCocco, M., Diamond, P., Maples, N. J., \& Davis, B. (2006). Use of environmental supports among patients with schizophrenia. Psychiatric Services, 57, 219-224.

Wexler, B., \& Bell, M. D. (2005). Cognitive remediation and vocational rehabilitation for schizophrenia. Schizophrenia Bulletin, 31(4), 931-941.

Weaverdyck, S. E. (2006). Assessing cognition of the person with cognitive impairment: Questions to ask. Cognitive Impairment Assessment Education Series, Session 2. Ann Arbor, MI: Michigan Alzheimer's Disease Research Center, University of Michigan. 